\title{
Molecular analysis of VcfQ protein involved in Vibrio cholerae type IV pilus biogenesis
}

\author{
Tomoko Miyazato, ${ }^{1}$ Claudia Toma, ${ }^{1}$ Noboru Nakasone, ${ }^{1}$ \\ Koichiro Yamamoto ${ }^{2}$ and Masaaki Iwanaga ${ }^{1}$

\begin{abstract}
${ }^{1}$ Department of Bacteriology, Faculty of Medicine, University of the Ryukyus, Uehara 207, Nishihara, Okinawa 903-0215, Japan

${ }^{2}$ Faculty of Health and Welfare Science, Okayama Prefectural University, Soja, Okayama 719-1197, Japan
\end{abstract}

Correspondence

Tomoko Miyazato

k008765@med.u-ryukyu.ac.jp

Received 9 May 2002

Accepted 23 December 2002

\begin{abstract}
The nucleotide sequence of an ORF ( $v c f Q$ ) within the type IV pilus gene cluster of Vibrio cholerae O34 strain NAGV14 was determined, thereby completing the sequence analysis of the structural operon. The $v c f Q$ gene showed homology to the $m s h Q$ gene of the mannose-sensitive haemagglutinin pilus gene cluster. The $v c f Q$ was 651 bp larger than $m s h Q$, and the $\mathrm{G}+\mathrm{C}$ content of the extra 651 bp portion (35.6 mol\%) was lower than that of the overall vcfQ gene $(42.5 \mathrm{~mol} \%)$. Except for the first 270 aa residues, the deduced amino acid sequence of VcfQ showed high homology to the MshQ protein. There was immunological cross-reaction between VcfQ and MshQ by Western blotting. Cell fractionation studies showed that $\mathrm{VcfQ}$ is located in both the inner and the outer membranes. Mutational analysis showed that $v c f Q$-deficient mutant expressed detectable levels of major pilin ( $\mathrm{CfA}$ ), but failed to assemble them into pili, indicating that $\mathrm{V} c f \mathrm{Q}$ is essential for pilus assembly. Colony-blotting analyses showed that the $\mathrm{N}$-terminal region of $v c f Q$ is variable in $V$. cholerae strains.
\end{abstract}

\section{INTRODUCTION}

Vibrio cholerae is known to be the causative agent of the acute gastrointestinal disease cholera. Strains of just two of the approximately 200 currently known O-antigen serogroups, $\mathrm{O} 1$ and O139, produce cholera toxin and cause cholera disease symptoms (Faruque et al., 1998; Yamai et al., 1997). However, sporadic cases of gastroenteritis including choleralike diarrhoea due to organisms with serogroups that are non-O1/non-O139 have been reported (Bhattacharya et al., 1998; Dalsgaard et al., 1999; Sharma et al., 1998). In the process of infection, the initial events are attachment of $V$. cholerae to the intestinal epithelium and colonization of the intestine by the organisms. Therefore, in studies of pathogenic mechanisms and in the efforts to develop vaccines, much attention has been focused on these events.

Type IV pili are flexible appendages which are found in a number of important pathogenic bacteria that affect humans and animals. Expression of type IV pili is associated with

\footnotetext{
Abbreviations: IM, inner membrane; MSHA, mannose-sensitive haemagglutinin; OM, outer membrane.

The GenBank/EMBL/DDBJ accession number for the $v c f Q$ sequence of V. cholerae strain NAGV14 is AB064660.

Figures showing the results of Western blotting analyses are available as supplementary data in JMM Online (http://jmm.sgmjournals.org).
}

colonization of epithelial cells, twitching motility of the organisms and biofilm formation on a variety of surfaces (Strom \& Lory, 1993). Several kinds of type IV pili of $V$. cholerae have been isolated, purified and characterized. The toxin-coregulated pilus (TCP) (Taylor et al., 1987) has been recently identified as a coat protein of a filamentous bacteriophage (Karaolis et al., 1999). Expression of a mannose-sensitive haemagglutinin (MSHA) pilus that promotes adherence to zooplankton and biofilm formation appears to be important for survival in an aquatic environment (Chiavelli et al., 2001; Watnick \& Kolter, 1999). The MSHA pilus is encoded in a locus that contains 16 genes organized in secretory and structural operons (Marsh \& Taylor, 1999). In the structural operon, five genes encode prepilin-like proteins and two encode putative outer-membrane (OM) proteins (Marsh \& Taylor, 1999).

In our previous studies, several kinds of pili of $V$. cholerae were purified and characterized (Iwanaga et al., 1989; Nakasone \& Iwanaga, 1990; Yamashiro et al., 1993, 1996). Among them, only the pili isolated from strain NAGV14 adhered to rabbit intestinal epithelium (Yamashiro et al., 1996). The major pilin structural gene of the NAGV14 pilus ( $v c f A)$ was highly homologous to the MSHA pilin gene in the $\mathrm{N}$-terminal region, but there was no homology in the Cterminal region (Kuroki et al., 2001). A subsequent study demonstrated that NAGV14 pilus gene cluster contained five 
ORFs that encoded prepilin-like proteins and corresponded to $m s h B, A, C, D, O$ (Toma et al., 2002). An ORF that encoded a putative OM protein and corresponded to $m s h P$ (Marsh \& Taylor, 1999; Toma et al., 2002) was also demonstrated. Considering this similarity of the both gene cluster organization in the two organisms, an orthologous protein (same functional protein) (Alm et al., 2000) of MshQ might exist in the NAGV14 pilus structural operon. In the present study, the $v c f Q$ gene encoding the putative MshQ-orthologous protein was investigated to clarify the gene function and to complete the nucleotide sequence of the NAGV14 pilus structural operon. The distribution of the gene in V. cholerae isolates was also studied.

\section{METHODS}

Bacterial strains, vector and media. The strains and plasmids used in this study are listed in Table 1. Other strains stocked in our laboratory were also used in colony blotting. The organisms were cultured in Luria-Bertani (LB) broth [ $1 \%$ tryptone (Difco), $0.5 \%$ yeast extract (Difco), $0.5 \% \mathrm{NaCl}$ (nacalai tesque)] or on LB agar plates that were supplemented, as necessary, with $100 \mu \mathrm{g}$ ampicillin $\mathrm{ml}^{-1}, 5 \mu \mathrm{g}$ kanamycin $\mathrm{ml}^{-1}$ and/or 5 or $25 \mu \mathrm{g}$ chloramphenicol $\mathrm{ml}^{-1}$.

DNA manipulation. Chromosomal DNA was isolated by using QIAGEN Genomic-tip (Qiagen). Plasmid DNA was isolated according to the method described by Birnboim \& Doly (1979) or by using Qiagen resin columns. Restriction enzyme digestion, ligation, gel electrophoresis, and transformation of DNA were carried out as described by Bagdasarian \& Bagdasarian (1994) or electroporation in a $0 \cdot 1 \mathrm{~cm}$ cuvette, using the Gene Pulser II Electroporation System (Bio-Rad) set at $0.75 \mathrm{kV}$. PCR products were purified on a GFX column (Amersham).
Sequencing and cloning of vcfQ. For sequencing and cloning of $v c f Q$, construction of the primers and PCR were performed as shown in Fig. 1. First, primers mshQ3 and mshQ4 were designed on the basis of the $m s h Q$ sequence previously reported by Marsh \& Taylor (1999), and the PCR product was named fragment-1. The nucleotide sequence of this fragment was determined, and new primers, mshQ10 and LA3 were constructed. For amplification of fragment-2, fragment-3 and fragment-4, primers DSV11, mreB, DSV13 and DSV8 were designed on the basis of previously reported sequences (Marsh \& Taylor, 1999; Toma et al., 2001). Fragment-5, which contains the complete $v c f Q$ gene, was amplified by using primers DSV13 and mshQ6 with KOD plus DNA polymerase (TOYOBO). Fragment- 5 was cloned into pCR 2.1 vector to obtain plasmid pCTQ20 after addition of $3^{\prime}$ A-overhangs which were necessary for TA cloning with Taq DNA polymerase (TOYOBO). All primers used in this study are presented in Table 2. Nucleotide sequencing was performed on both strands with Big Dye Terminator Cycle Sequencing FS Ready Reaction kits (Applied Biosystems) and analysed with an ABI PRISM 310 Genetic Analyzer (Perkin-Elmer). The program BLAST 1.4.9 was used to search for homologous sequences in the database.

Cloning of $\boldsymbol{m s h} \boldsymbol{Q}$. The same protocol used for cloning of $v c f Q$ was used to clone the $m s h Q$ gene from $V$. cholerae $\mathrm{O} 1$ strain $86 \mathrm{~B} 3$. The complete $m s h Q$ gene was amplified by using primers QCE1 and mshQ6. The PCR product was cloned into PCR 2.1 vector and the resulting plasmid (pQ28-4) was used to transform Escherichia coli XL-1 Blue. E. coli XL-1 Blue harbouring pQ28-4 was used for immunological detection of MshQ.

Cloning the $\mathrm{C}$-terminal region of vcf $Q$ into the expression vector. The C-terminal region (VcfQT) of $v c f Q$ was amplified by PCR using primers nagQ15 and nagQ18. The PCR product was digested with BamHI and SmaI and cloned into the corresponding sites of pQE30 vector. The recombinant plasmid (pTM2) was transformed into E. coli M15.

Table 1. Strains and plasmids used in this study

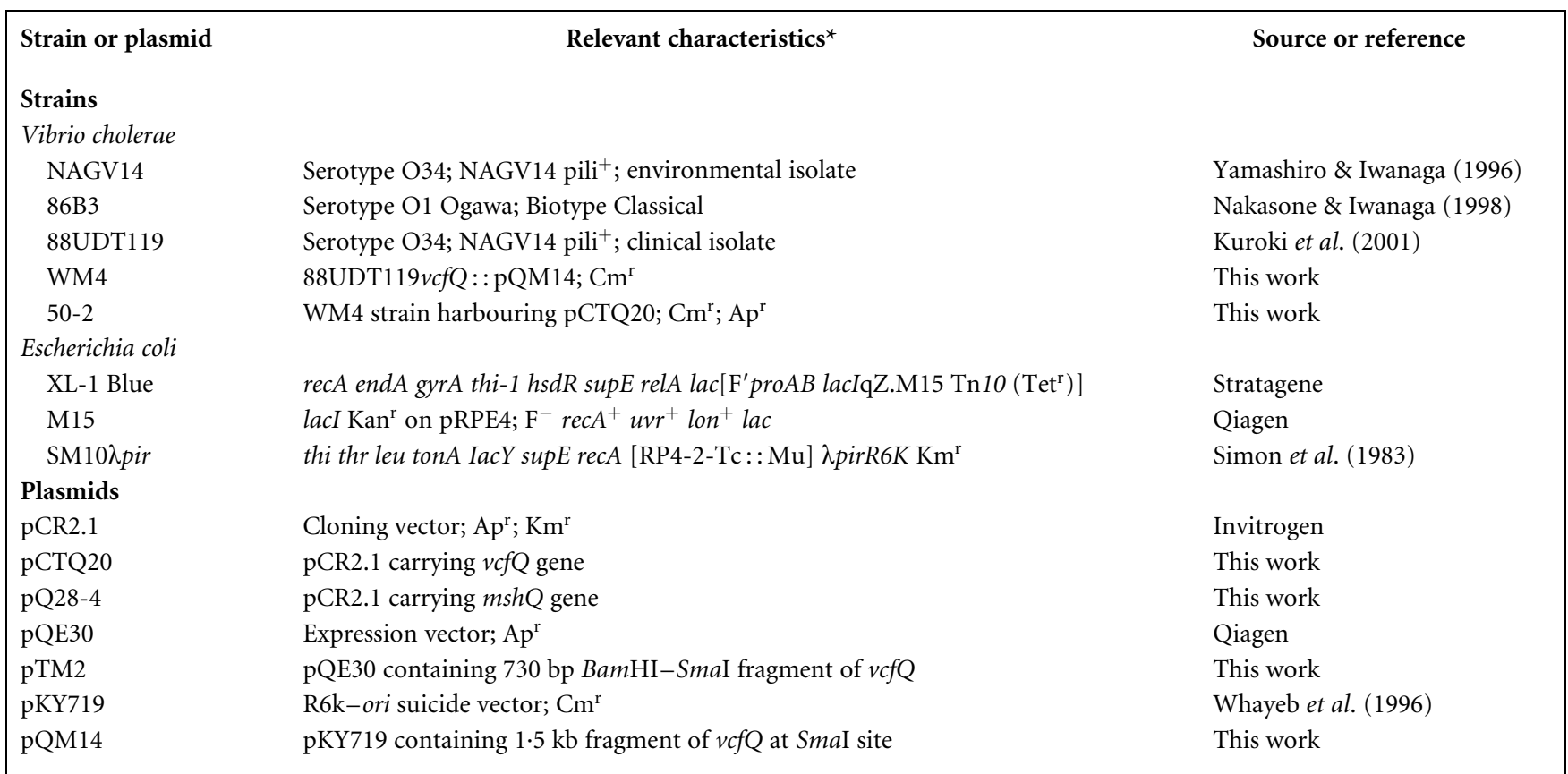

${ }^{\star} \mathrm{Cm}^{\mathrm{r}}$, chloramphenicol resistance; $\mathrm{Ap}^{\mathrm{r}}$, ampicillin resistance; $\mathrm{Km}^{\mathrm{r}}$, kanamycin resistance. 
$1 \mathrm{kbp}$

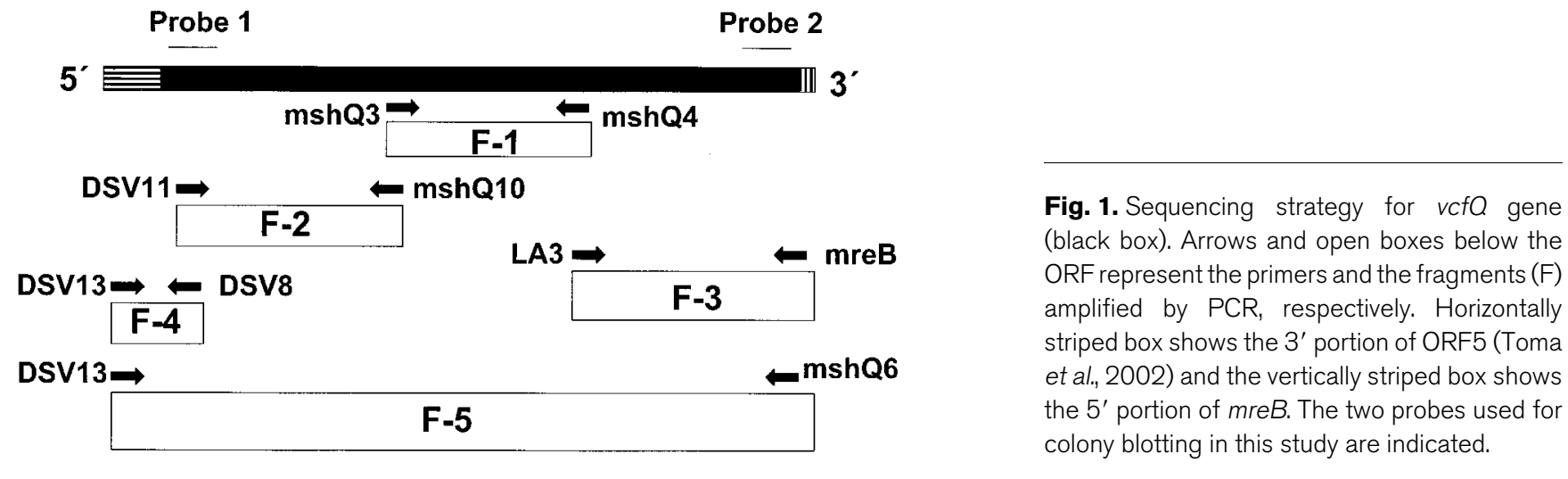

Table 2. Oligonucleotide primers used in PCR, protein expression or probes

All primers were designed in this study. Underlined letters indicate restriction endonuclease sites. F, Forward primer; R, reverse primer.

\begin{tabular}{|llcc|}
\hline $\begin{array}{l}\text { Primer or } \\
\text { probe }\end{array}$ & \multicolumn{1}{c|}{ Sequence $\left(\mathbf{5}^{\prime} \rightarrow \mathbf{3}^{\prime}\right)$} & Positions or locus & \\
\hline mshQ3(F) & TCCGAGCTCGGTGGCTCTCTAGGTTATGC & & Sequencing \\
mshQ4(R) & CGGGGTACCGGCTGCACCAGAGTGTGTTG & $2510-2529^{*}$ & Sequencing \\
mshQ10(R) & GACTTGACTGGCATTACGGG & $3995-4014^{*}$ & Sequencing \\
LA3(F) & AGTGATGGTCAATGCCCTGC & $1826-1845$ & Sequencing \\
DSV11(F) & ATTGAGTCCACGCTTGTGGC & $2818-2837$ & Sequencing, probe 1 \\
mreB(R) & GGCGGTACCTAAATCGATCG & $2294-2313 \dagger$ & Sequencing \\
DSV13(F) & CTGGGGGCTTTAGATAAT & $5369-5388^{*}$ & Sequencing \\
DSV8(R) & GTGTCCTAGTTAGCTTGTAG & $2001-2020 \dagger$ & Sequencing \\
mshQ6(R) & TTAATTCGATTCGCGCCACC & $1-20$ & Sequencing, probe 2 \\
QCE1(F) & GAGATTGGAGCTTACAACGC & $5142-5161^{*}$ & Cloning \\
nagQ15(F) & CCGGGATCCGATTTCTCTTTGGTCGCCCC & $1361-1380^{*}$ & Expression \\
nagQ18(R) & TCCCCCGGGGTAAAGCCTGATATCGCAGG & $3100-3119$ & Expression \\
PUCF(F) & GAATTCGAGCTCGGTACCC & $3817-3836$ & Mutant confirmation \\
nagQ7(R) & CAAGGATCCCAACGCCATTCCTGTGGAGG & $1228-1247$ & Mutant confirmation \\
nagQ10(R) & CGTCACTGGATTATTCGCTG & $284-303$ & Probe 1 \\
nagQ11(F) & TTCCCACCAATTGATGGAGC & $3658-3677$ & Probe 2 \\
\hline
\end{tabular}

${ }^{\star}$ GenBank accession no. AF079234.

$\dagger$ GenBank accession no. AB049152.

Expression and purification of recombinant His-tagged protein. VcfQT was purified from E. coli M15 harbouring plasmid pTM2 as a (His) ${ }_{6}$-tagged recombinant protein. Overnight culture of the recombinant organism was diluted 1:50 in LB medium (containing ampicillin and kanamycin) and induced with $1 \mathrm{mM}$ IPTG. The harvested cells were dissolved in start buffer ( $8 \mathrm{M}$ urea, $0.1 \mathrm{M} \mathrm{NaH}_{2} \mathrm{PO}_{4}, 0.01 \mathrm{M}$ Tris, 10 $\mathrm{mM}$ imidazole, $\mathrm{pH} 8 \cdot 0$ ). The soluble fraction was collected and purified using a His Trap nickel column (Amersham) using $500 \mathrm{mM}$ imidazole phosphate buffer as the elution buffer.

Antiserum preparation. A rabbit was immunized with $87.5 \mu \mathrm{g}$ purified VcfQT protein in Freund's complete adjuvant. The animal was boosted three times with the same antigen in Freund's incomplete adjuvant at 2-week intervals.

SDS-PAGE and Western blotting analysis. SDS-PAGE and
Western blotting were carried out according to the methods of Laemmli (1970) and Towbin et al. (1979), respectively. Pre-stained molecular markers (New England Biolabs) were used as size standards.

Cellular fractionation. Cellular fractionation was accomplished by a modification of the method of Ramer et al. (2002). V. cholerae 88UDT119 was cultured in $500 \mathrm{ml} \mathrm{LB}$ broth at $37^{\circ} \mathrm{C}$ overnight with shaking. The harvested cells were suspended in $8 \mathrm{ml} 1 \mathrm{M}$ sucrose in 30 $\mathrm{mM}$ Tris/ $\mathrm{HCl}(\mathrm{pH} 8 \cdot 0)$, to which $80 \mu \mathrm{l} 0.5 \mathrm{M}$ EDTA and $80 \mu \mathrm{l}$ of a $20 \mathrm{mg} \mathrm{ml}^{-1}$ lysozyme solution were added. The suspension was then incubated on ice for $40 \mathrm{~min} . \mathrm{MgCl}_{2}$ was added to a final concentration of $75 \mathrm{mM}$. The cells were centrifuged at $15000 \mathrm{~g}$ for $30 \mathrm{~min}$ and the resulting supernatant was collected as the periplasmic fraction. The cell

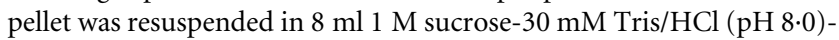
$75 \mathrm{mM} \mathrm{MgCl}_{2}$. The resulting suspension was sonicated four times ( 1 min each) and then subjected to two rounds of freezing and thawing. 
The unbroken cells were removed by centrifugation at $5000 \mathrm{~g}$ for $10 \mathrm{~min}$ and supernatant was subjected to further centrifugation at $113000 \mathrm{~g}$ for $1 \mathrm{~h}$. The resulting supernatant was termed the cytoplasmic fraction. The pellet was resuspended in $1 \mathrm{ml} 10 \mathrm{mM}$ PBS, and then $100 \mu \mathrm{l} 10 \%$ Sarkosyl was added. The suspension was treated with a glass homogenizer, and after centrifugation at $113000 \mathrm{~g}$ for $1 \mathrm{~h}$, the Sarkosylsoluble fraction was termed the inner-membrane (IM) fraction. The pellet resuspended in $500 \mu \mathrm{l}$ PBS was termed the OM fraction.

Construction of vcfQ insertion mutant. To confirm that VcfQ is required for type IV pilus biogenesis, the $v c f Q$ gene was disrupted by insertion of a suicide plasmid. A $1.5 \mathrm{~kb}$ central fragment amplified by PCR with primer mshQ3 and mshQ4 (fragment-1, Fig. 1) was cloned in the suicide plasmid pKY719 (Whayeb et al., 1996) to obtain plasmid pQM14. Bacterial conjugation was accomplished by mating E. coli SM10 $\lambda$ pir bearing pQM14 as a donor and $V$. cholerae 88UDT119 as recipient. The $v c f Q$ exconjugants were selected on TCBS agar containing $5 \mu \mathrm{g}$ chloramphenicol $\mathrm{ml}^{-1}$. The $v c f Q$ plasmid insertion mutant, WM4, was identified by PCR using primer pUCF, which is specific for the multiple cloning site of pKY719, and primer nagQ7, which is specific for sequences upstream of primer mshQ3. To complement the $v c f Q$ mutation in WM4, pCTQ20, containing $v c f Q$, was electroporated into WM4 to obtain strain 50-2 (Table 1).

Crude type IV pili preparation. Crude NAGV14 pili were prepared as described previously (Nakasone \& Iwanaga, 1990).

Colony blotting. A single colony was picked and placed onto a nylon membrane Hybond- $\mathrm{N}^{+}$(Amersham). Alkaline lysis of the cells was performed and then probed with peroxidase-labelled PCR-amplified DNA fragments (Fig. 1). Hybridization was performed overnight at $42{ }^{\circ} \mathrm{C}$, and high-stringency washes were performed before detection with the ECL direct nucleic acid labelling and detection system (Amersham).

\section{RESULTS AND DISCUSSION}

\section{Nucleotide sequence analysis}

The nucleotide sequences of fragments-1, 2, 3 and 4 (Fig. 1) were determined. To confirm the sequence, fragment-5 (Fig. 1 ) was cloned and sequenced. The sequence of fragment- 5 contained a complete ORF $(v c f Q)$ of $4410 \mathrm{bp}$. The deduced amino acid sequence revealed that $v c f Q$ encoded a 1470 aa residue with a calculated molecular mass of $157.9 \mathrm{kDa}$ and an isoelectric point of $4 \cdot 77$. VcfQ is predicted by PSORT analysis (Nakai \& Kanehisa, 1991) to be an OM protein with a potential signal sequence of 19 aa.

Homology searches comparing the nucleotide sequence of $v c f Q$ with other sequences indicated that portions of $v c f Q$ showed homology to those of $m s h Q$; however, $v c f Q$ was $651 \mathrm{bp}$ larger than $m s h Q$. The size difference between $v c f Q$ and $m s h Q$ might be explained by the difference of $\mathrm{G}+\mathrm{C}$ content of the extra $651 \mathrm{bp}$ region. The $\mathrm{G}+\mathrm{C}$ content of $v c f \mathrm{Q}$ $(42.5 \mathrm{~mol} \%)$ and $m s h Q(45 \mathrm{~mol} \%)$ are similar; however, the $\mathrm{G}+\mathrm{C}$ content was lower $(35.6 \mathrm{~mol} \%)$ in the extra region. This may indicate that the region was introduced into the NAGV14 strain from some other organism since different $\mathrm{G}+\mathrm{C}$ content is a reliable indicator of horizontal transfer (Lawrence \& Ochman, 1998). As in V. cholerae O1, a portion of the $m r e B$ gene (Marsh \& Taylor, 1999) was also found downstream of $v c f Q$.

BLASTP analysis of the deduced amino acid sequence revealed that the first 270 residues of VcfQ had no similarity with any known protein. However, high homology was detected between the VcfQ (residues 271-1470) and MshQ (residues 43-1253) (61\% identity, $70 \%$ similarity) (Fig. 2). This homology was supported by the immunological crossreaction found in Western blotting using VcfQ specific antiserum (anti-VcfQ antiserum) and whole-cell lysates of E. coli harbouring pCTQ20 or pQ28-4 (see gel in the supplementary data system in JMM Online; http:// jmm.sgmjournals.org). By determining the $v c f Q$ gene nucleotide sequence, we completed the genetic sequence analysis of the NAGV14 pilus structural operon. The $v c f Q$ gene was identified as the last gene of the pilus operon.

\section{Localization of VcfQ}

Many Gram-negative bacteria express type IV pili on their surface; however, the mechanism of type IV pili biogenesis is poorly understood. The genes required for type IV pili biogenesis encode major pilin, minor pilin(s) and IM or OM proteins (Marsh \& Taylor, 1999). The predicted amino acid sequence of $v c f Q$ showed that $\mathrm{VcfQ}$ might be an OM protein. To investigate this possibility, cell fractions prepared from $V$. cholerae 88UDT119 were analysed by Western blotting with anti-VcfQ antiserum. As shown in the gel in the supplementary data system in JMM Online (http:// jmm.sgmjournals.org), anti-VcfQT antiserum recognized a $157 \mathrm{kDa}$ VcfQ protein in OM fraction of 88UDT119. The result shows that this protein is $\mathrm{OM}$-associated. However, a detectable level of $\mathrm{VcfQ}$ was also found in the IM. Therefore, VcfQ might coalesce into an assembly complex by anchoring to IM and thus may articulate between the proteins in the IM and OM components. The presence of VcfQ in the IM and OM could be explained by disruption of this complex during cell fractionation. In E. coli type IV pilus (bundle-forming pilus, BFP), the assembly complex has been reported to consist of IM, OM and periplasmic components. One of

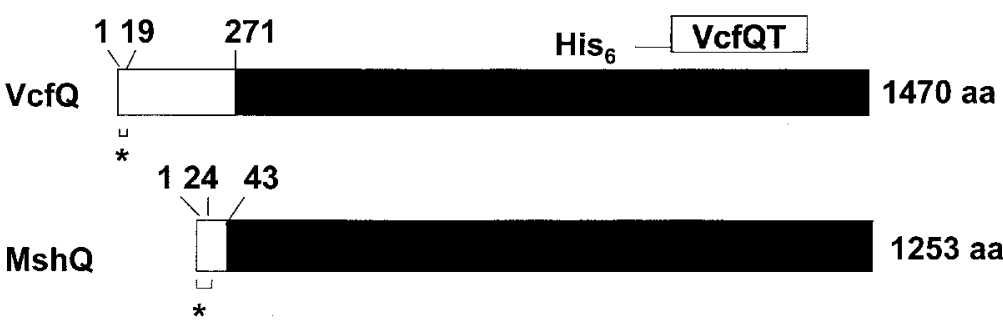

Fig. 2. Comparison of the deduced amino acid sequences of $\mathrm{VcfO}$ and MshO. Black boxes represent homologous regions of $\mathrm{VcfO}$ and MshQ. White boxes represent variable regions. Numbers show amino acid position relative to the methionine of $\mathrm{VcfO}$ and $\mathrm{MshO}$ taken as 1. Asterisks show signal sequence. The location of recombinant protein VcfOT is indicated. 
these components, BfpL, was also shown to be associated with the IM and OM fractions and essential for BFP biogenesis (Ramer et al., 2002).

\section{Phenotype of vcfQ-deficient mutant}

To assess the role of $v c f Q$ in type IV pilus biogenesis, we constructed a $V$. cholerae 88UDT119 strain containing insertion mutation in the $v c f Q$ gene (WM4). Western blotting analysis using whole-cell lysates of WM4 with an anti-VcfA antiserum (Toma et al., 2002) revealed that the WM4 did produce normal levels of $v c f A$ (see gel in the supplementary data system in JMM Online; http:// jmm.sgmjournals.org). Multiple bands that appeared at 20, 21 and $22 \mathrm{kDa}$ were likely due to the post-translational modification of pili as previously described by Kuroki et al. (2001). The type IV pilus of Pseudomonas aeruginosa and Neisseria meningitidis have been reported to be post-translationally modified by glycans (Castric, 1995; Power et al., 2000). Despite the presence of pilin, no pilus was detected in the crude pili fraction of WM4. Therefore, WM4 failed to assemble VcfA into pili. The complemented strain, 50-2, produced normal levels of pilin and extracellular pili. These results indicate that VcfQ is essential for pilus assembly but not for expression of VcfA.

\section{Colony-blotting analysis}

To investigate the conservation of $v c f Q$ and $m s h Q$ in various strains, 93 strains of $V$. cholerae (Table 3 ) were tested by colony blotting using probe 1 and probe 2 (Table 2, Fig. 1). The results presented in Table 3 show that only one of the 25 $V$. cholerae $\mathrm{O} 1$ strains tested and none of the $18 \mathrm{~V}$. cholerae O139 strains tested gave a positive signal with probe 1, which anneals to the $\mathrm{N}$-terminal region of $v c f Q$. For $V$. cholerae nonO1/non-O139, $20 \%$ (10 of 50) of the strains tested gave positive signals with probe 1 . In contrast, $92 \%$ (23 of 25 ) of $V$. cholerae $\mathrm{O} 1$ strains, $100 \%$ (18 of 18 ) of $V$. cholerae $\mathrm{O} 139$ strains and $98 \%$ ( 49 of 50) of V. cholerae non-O1/non-O139 strains tested produced strong signals with probe 2, which anneals to the C-terminal region of $v c f Q$ and $m s h Q$. We previously demonstrated that the NAGV14 pilus and MSHA pilus gene clusters are different in the C-terminal region of the gene $(v c f A)$ encoding the major pilin subunit (Kuroki et al., 2001). In this study we elucidated that the N-terminal region of the gene $(v c f Q)$ encoding the $\mathrm{OM}$-associated protein is also variable.

Table 3. Conservation of $v c f Q$ and $m s h Q$

\begin{tabular}{|lccc|}
\hline Serogroup & No. strains & \multicolumn{2}{c|}{$\begin{array}{c}\text { No. (\%) strains positive with } \\
\text { probe: }\end{array}$} \\
\cline { 2 - 4 } & & $\mathbf{1}$ & $\mathbf{2}$ \\
\hline O1 & & $1(4)$ & $23(92)$ \\
O139 & 25 & $0(0)$ & $18(100)$ \\
Others & 18 & $10(20)$ & $49(98)$ \\
\hline
\end{tabular}

One possible way for $V$. cholerae to cause epidemic disease is adhesion to the zooplankton using type IV pili and survival in the environment (Chiavelli et al., 2001). V. cholerae has to assemble the pili under many environmental conditions. Thus, the molecular variation detected here in the gene encoding an OM protein included in the type IV pilus gene cluster might be required for type IV pilus biogenesis.

\section{ACKNOWLEDGEMENTS}

This work was supported by a Grant-in-Aid for Scientific Research from the Ministry of Education, Culture, Sports, Science and Technology, Japan.

\section{REFERENCES}

Alm, R. A., Bina, J., Andrews, B. M., Doig, P., Hancock, R. E. W. \& Trust, T. J. (2000). Comparative genomics of Helicobacter pylori: analysis of the outer membrane protein families. Infect Immun 68, 4155-4168.

Bagdasarian, M. \& Bagdasarian, M. M. (1994). Gene cloning and expression. In Methods for General and Molecular Bacteriology, pp. 409-412. Edited by P. Gerhardt, R. G. E. Murray, W. A. Wood \& N. R. Krieg. Washington, DC: American Society for Microbiology.

Bhattacharya, M. K., Dutta, D., Bhattacharya, S. K. \& 7 other authors (1998). Association of a disease approximating cholera caused by Vibrio cholerae of serogroups other than O1 and O139. Epidemiol Infect 120, $1-5$.

Birnboim, H. C. \& Doly, J. (1979). A rapid alkaline extraction procedure for screening recombinant plasmid DNA. Nucleic Acids Res 7, 1513-1523.

Castric, P. (1995). pilO, a gene required for glycosylation of Pseudomonas aeruginosa 1244 pilin. Microbiology 141, 1247-1254.

Chiavelli, D. A., Marsh, J. W. \& Taylor, R. K. (2001). The mannosesensitive hemagglutinin of Vibrio cholerae promotes adherence to zooplankton. Appl Environ Microbiol 67, 3220-3225.

Dalsgaard, A., Forslund, A., Bodhidatta, L., Serichantalergs, O., Pitarangsi, C., Pang, L., Shimada, T. \& Echeverria, P. (1999). A high proportion of Vibrio cholerae strains isolated from children with diarrhea in Bangkok, Thailand are multiple antibiotic resistant and belong to heterogeneous non-O1, non-O139 O-serotypes. Epidemiol Infect 122, 217-226.

Faruque, S. M., Albert, M. J. \& Mekalanos, J. J. (1998). Epidemiology, genetics, and ecology of toxigenic Vibrio cholerae. Microbiol Mol Biol Rev 62, 1301-1314.

Iwanaga, M., Nakasone, N. \& Ehara, M. (1989). A comparative study on adhesive and non-adhesive strains. Microbiol Immunol 33, 1-9.

Karaolis, D. K. R., Somara, S., Maneval, D. R., Jr, Johnson, J. A. \& Kaper, J. B. (1999). A bacteriophage encoding a pathogenicity island, a type IV pilus and a phage receptor in cholera bacteria. Nature 399, 375-379.

Kuroki, H., Toma, C., Nakasone, N., Yamashiro, T. \& Iwanaga, M. (2001). Gene analysis of Vibrio cholerae NAGV14 pilus and its distribution. Microbiol Immunol 45, 417-424.

Laemmli, U. K. (1970). Cleavage of structural proteins during the assembly of the head of bacteriophage T4. Nature 227, 680-685.

Lawrence, J. G. \& Ochman, H. (1998). Molecular archaeology of the Escherichia coli genome. Proc Natl Acad Sci U S A 95, 9413-9417.

Marsh, J. W. \& Taylor, R. K. (1999). Genetic and transcriptional analysis of the Vibrio cholerae mannose-sensitive hemagglutinin type 4 pilus gene locus. J Bacteriol 181, 1110-1117.

Nakai, K. \& Kanehisa, M. (1991). Expert system for predicting protein localization sites in gram-negative bacteria. Proteins 11, 95-110. 
Nakasone, N. \& Iwanaga, M. (1990). Pili of Vibrio cholerae non-O1. Infect Immun 58, 1640-1646.

Nakasone, N. \& Iwanaga, M. (1998). Characterization of outer membrane protein OmpU of Vibrio cholerae O1. Infect Immun 66, $4726-4728$.

Power, P. M., Roddam, L. F., Dieckelmann, M., Srikhanta, Y. N., Tan, Y. C., Berrington, A. W. \& Jennings, M. P. (2002). Genetic characterization of pilin glycosylation in Neisseria meningitidis. Microbiology 146, 967-979.

Ramer, S. W., Schoolink, G. K., Wu, C. Y., Hwang, J., Schmidt, S. A. \& Bieber, D. (2002). The type IV pilus assembly complex: biogenic interactions among the bundle-forming pilus proteins of enteropathogenic Escherichia coli. J Bacteriol 184, 3457-3465.

Sharma, C., Thungapathra, M., Ghosh, A. \& 11 other authors (1998). Molecular analysis of non-O1, non-O139 Vibrio cholerae associated with an unusual upsurge in the incidence of cholera-like disease in Calcutta, India. J Clin Microbiol 36, 756-763.

Simon, R., Priefer, U. \& Pühler, A. (1983). A broad host range mobilization system for in vivo genetic engineering: transposon mutagenesis in gram negative bacteria. Bio-technology 11, 784-791.

Strom, M. S. \& Lory, S. (1993). Structure-function and biogenesis of the type IV pili. Annu Rev Microbiol 47, 565-596.
Taylor, R. K., Miller, V. L., Furlong, D. B. \& Mekalanos, J. J. (1987). Use of phoA gene fusions to identify a pilus colonization factor coordinately regulated with cholerae toxin. Proc Natl Acad Sci U S A 84, 2833-2837.

Toma, C., Kuroki, H., Nakasone, N., Ehara, M. \& Iwanaga, M. (2002). Minor pilin subunits are conserved in Vibrio cholerae type IV pili. FEMS Immunol Med Microbiol 33, 35-40.

Towbin, H., Staehelin, T. \& Gordon, J. (1979). Electrophoretic transfer of proteins from polyacrylamide gels to nitrocellulose sheets: procedure and some applications. Proc Natl Acad Sci U S A 76, 4350-4354.

Watnick, P. I. \& Kolter, R. (1999). Steps in the development of a Vibrio cholerae El Tor biofilm. Mol Microbiol 34, 586-595.

Whayeb, L. A., Yamamoto, K., Castillo, M. E. Y., Tojo, H. \& Honda, T. (1996). Lysophospholipase $\mathrm{L}_{2}$ of Vibrio cholerae $\mathrm{O} 1$ affects cholera toxin production. FEMS Immunol Med Microbiol 15, 9-15.

Yamai, S., Okitsu, T., Shimada, T. \& Katsube, Y. (1997). Distribution of serogroups Vibrio cholerae non-O1 non-O139 with specific reference to their ability to produce cholera toxin and additional of novel serogroups. Jpn J Assoc Infect Dis 71, 1037-1045.

Yamashiro, T. \& Iwanaga, M. (1996). Purification and characterization of a pilus of a Vibrio cholerae strain: a possible colonization factor. Infect Immun 64, 5233-5238.

Yamashiro, T., Nakasone, N. \& Iwanaga, M. (1993). Purification and characterization of pili of a Vibrio cholerae non-O1 strain. Infect Immun 61, 5398-5400. 